\title{
Churg-Strauss syndrome with broad spectrum clinical presentations: a report of 3 cases
}

\author{
B. Mokri, ${ }^{\text {S.F. Tafti }}{ }^{2}$ and F. Talischi ${ }^{2}$
}

\section{Introduction}

Churg-Strauss syndrome (CSS) is a rare systemic vasculitis characterized by hypereosinophilia, necrotizing vasculitis with granulomas of extravascular eosinophils and asthma history [1]. CSS has also been shown to be a pathological syndrome of angiitis and allergic granulomatosis [2]. Three principal classifications are described:

- Lanham's criteria which depend upon clinical aspects [1].

- Chapel Hill Consensus Conference [3].

- The American College of Rheumatology that has suggested 6 criteria from which 4 are required for a diagnosis of CSS with $99.7 \%$ specificity and $85 \%$ sensitivity. The 6 criteria include: asthma, eosinophilia $>10 \%$, paranasal sinusitis, vasculitis based on histology, and mononeuritis multiplex [4].

Vasculitis lesions of CSS on histological examination are not typically seen in biopsies and are not specific for CSS; at the same time their absence is not cause to reject a diagnosis of CSS [5].

It is important to differentiate CSS from other systemic vasculitis because of the association between start of treatment and CSS prognosis [6,7].

In the present study, we report 3 CSS cases with diverse clinical presentations to investigate if a clinical approach and history of the patient could be diagnostic for CSS without invasive procedures such as histological confirmation by biopsy. We also sought to assess whether the absence of leukocytosis and eosinophilia could allow a diagnosis of CSS to be rejected.

\section{Case reports}

\section{Case 1}

A 19-year-old woman was admitted to our hospital, Masih Daneshvari Hospital, in November 2008 with pseudo common cold symptoms, myalgia, nasal congestion and urticarial rashes. She was discharged on steroid therapy prescribed following skin biopsy reports of urticarial vasculitis. Two weeks later, she returned to the centre with dyspnoea, wheezing and severe rhinorrhoea. On general examination, severe nasal polyps and bilateral wheezing on breathing were detected.

Laboratory findings revealed eosinophilia (blood eosinophil count of $33 \%$ ), white blood cell (WBC) count $11500 / \mathrm{mm}^{3}$ and erythrocyte sedimentation rate (ESR) of $32 \mathrm{~mm} / \mathrm{h}$. Other laboratory findings were normal. Biopsy of the nasal mucosashowed eosinophilic infiltration. Computer tomography (CT) of the sinuses showed opacities in all sinuses. Nasal polypectomy was carried out and revealed vasculitis with perivascular granuloma. Chest spiral CT before hospitalization in this centre revealed patchy infiltrates.

The patient was diagnosed with CSS and prescribed prednisolone therapy (50 mg/day). She became symptom-free after 10 days. Up to May 2010, the patient had not been rehospitalized and now uses prednisolone $(5 \mathrm{mg} /$ day 3 days a week and $2.5 \mathrm{mg}$ /day 4 days a week) and azathioprine ( $5 \mathrm{mg} /$ day).

\section{Case 2}

A 55-year-old man with a 3-year history of dyspnoea, wheezing and recurrent sinusitis was hospitalized in June 2002 with haemoptysis. The patient had received inhaled bronchodilator drugs and steroids before hospital admission. He had had a nasal polypectomy 7 years before. On clinical examination, bilateral expiratory wheezing was noted in chest auscultation. Chest X-ray showed bilateral diffused alveolar patches.

In laboratory tests, blood eosinophilia (eosinophil count of 30\%), WBC $22000 / \mathrm{mm}^{3}$ and ESR $45 \mathrm{~mm} / \mathrm{h}$ were found. Laboratory indices for autoimmune diseases were absent. In addition, a sputum culture test was negative on 3 occasions. On the third hospital day, skin rashes appeared on the front of his legs. Skin biopsy was done and showed perivascular infiltration of neutrophils and eosinophils with fibrinoid necrosis. He was diagnosed with CSS and treated with methyl prednisolone pulse therapy (1 g) followed by oral steroids $(50 \mathrm{mg} /$ day) for 3 weeks. The patient died 4 years after discharge from hospital due to CSS recurrence. 


\section{Case 3}

A 45-year-old woman was hospitalized in our medical centre in January 2002 because of maculopapular rashes on her lower extremities, fever, dyspnoea and decreased muscle power in the right leg that started a month before admission. She had a 1-year history of bronchial asthma diagnosed by dyspnoea, cough and increased sputum production. She was taking corticosteroid, methotrexate and zafirleukast, which had not resulted in improvement. Physical examination revealed respiratory distress, skin rashes, expiratory generalized wheezing in breathing and a neurological finding of mononeuritis multiplex that was confirmed by electromyography and nerve conduction studies. Laboratory findings showed WBC count of $20100 /$ $\mathrm{mm}^{3}$ and with eosinophil count of $10 \%$. The other findings and collagen vascular tests were normal. Bilateral infiltration in the lower fields of the lungs was seen on chest X-ray. Water's view X-ray of the skull demonstrated pansinusitis. Skin biopsyindicated perivascular infiltration of lymphocytes and eosinophils and also endothelial cell proliferation with a microgranuloma. Bronchoalveolar lavage revealed eosinophilia in bronchial secretion. She was diagnosed as CSS and the signs remitted after 2 weeks of treatment with corticosteroid. The patient has been free of symptoms with no recurrence of CSS to May 2010.

\section{Discussion}

CSS is a rare systemic small-vessel necrotizing vasculitis and multiorgan disorder characterized by asthma, extravascular necrotizing granulomas, lung infiltrates and hypereosinophilia. Asthma and hypereosinophilia are the main features of CSS in most cases. Allergic rhinitis, maxillary sinusitis, and sinus polyposis are seen in $70 \%$ of patients. Antineutrophil cytoplasm antibodies (ANCA) are found in a third to a half of cases. General symptoms associated with pulmonary infiltrates, peripheral neuropathy, usually mononeuritis multiplex, and skin, gastrointestinal tract and cardiac involvement are frequent.

The pathophysiology of CSS suggests that ANCA are probably responsible for CSS vasculitic manifestations, while eosinophil infiltration and related cytotoxicity could be involved in cardiomyopathy.

The factors associated with poor prognosis of CSS are renal failure, severe gastrointestinal tract involvement, cardiomyopathy and central nervous systems involvement $[8,9]$.

The first line therapy for CSS should include a high dose of a corticosteroid. Immunosuppressive cytotoxic therapy is indicated if corticosteroid therapy is insufficient to control the disease or when the patient has factors associated with poor prognosis. Intravenous immunoglobulin therapy can be used as a second line in cases resistant to the standard treatment, such as cases of neuropathy or cardiomyopathy. Following treatment of CSS, the outcome is good with a 5-year survival of $90 \%$, but asthma often remains [10].

CSS is distinguished by the presence of asthma history, blood eosinophilia and necrotizing vasculitis $[1,11,12]$. In the absence of any biopsy or bronchoalveolar lavage fluid, other disorders causing pulmonary eosinophilic infiltrates should be considered and the reasons for eosinophilic lung disease should sought. Based on the classification by Crofton et al., which was extended by Davis and Allen, Löffler syndrome, chronic and acute eosinophilic pneumonia, idiopathic hypereosinophilic syndrome, asthma, certain parasitic infections, drug reactions, bronchocentric granulomatosis and allergic bronchopulmonary aspergillosis (ABPA) are CSS differential diagnosis.

The most common differential diagnosis of CSS is ABPA which appears with central bronchiectasis and $\operatorname{IgE}$ increase. Absence of multisystem disease differentiates ABPA from CSS [13-15].

Chronic eosinophilic pneumonia, another differential diagnosis of CSS, presents with cough, fever, weight loss and dyspnoea. Most patients have asthma. Elevation of eosinophil count in peripheral blood and bronchoalveolar lavage fluid is seen in $90 \%$ of patients. The absence of multisystem involvement and radiographic findings showing peripheral infiltrates differentiates chronic eosinophilic pneumonia from CSS $[16,17]$.

Another principal differential diagnosis of CSS is idiopathic hypereosinophilic syndrome. Similar to CSS, idiopathic hypereosinophilic syndrome causes multiorgan involvement, but is can be distinguished by a peripheral blood eosinophil count $>1500 / \mathrm{mm}^{3}$ for more than 6 months [18]. Therefore, clinical pictures, laboratory findings and course of illness can differentiate idiopathic hypereosinophilic syndrome and CSS [7].

Normal cardiac size, absence of pleural effusion and nonexistense of irreversible pulmonary complications such as honeycomb fibrosis, traction bronchiectasis and architectural distortion in high-resolution CT can lead to CSS diagnosis. Also, arthropathies, ophtalmopathies and hepatobiliary involvement are very rare in CSS versus other vasculitis disorders $[7,19]$. Some studies have reported that CSS can appear following the use of leukotriene-modifying drugs, corticosteroid withdrawal and history of polypectomy and vaccination desensitization, but these criteria cannot be considered diagnostic [20-22].

Recent reports of CSS suggest an increase in CSS incidence in the Islamic Republic of Iran; however the exact incidence of CSS is not known [23-25].

All 3 of our patients had a history of severe asthma and were found to have eosinophilia. In the first patietn, nasal polyp, urticarial skin lesions and eosinophilic infiltrations in polyps as 
well as vasculitis with granuloma in the nasal mucosa were found and led to a diagnosis of CSS. In the second patient, haemoptysis, skin rashes on the legs, pulmonary infiltration, perivascular fibrinoid necrosis, neutrophil and eosinophil infiltration led to CSS diagnosis. In the last patient, skin rashes, fever, right foot weakness with electromyography and nerve conduction velocity findings were diagnostic manifestations for CSS.

As can be seen with our cases, CSS can present with a broad range of clinical features. We carried out clinical and laboratory investigations for our patients which were appropriate and helpful for diagnosis of CSS; however excluding other causes of eosinophilia and establishing vasculitis in the patients was necessary to distinguish from other disorders and diagnose CSS.

Therefore, we believe CSS can be diagnosed by an accurate history, clinical examination and laboratory tests with no need for invasive procedures such as lung biopsy.
Our conclusion is based on only 3 cases and the diagnosis was not confirmed by biopsy/bronchoalveolar lavage fluid. However, it suggests that CSS could be diagnosed without invasive tests, which would be beneficial for patients and reduce costs for hospitals. There is a need therefore for further research with a large sample and comparing with the gold standard to establish whether CSS can be reliably diagnosed without the need for lung biopsy.

\section{References}

1. Lanham JG et al. Systemic vasculitis with asthma and eosinophilia: a clinical approach to the. Medicine, 1984, 63:65-81.

2. Churg J, Strauss L. Allergic granulomatosis, allergic angiitis, and periarteritis nodosa. American Journal of Pathology, 1951, 27:277-301.

3. Jennette JC et al. Nomenclature of systemic vasculitides. Proposal of an international consensus conference. Arthritis and Rheumatism, 1994, 37:187-192.

4. Masi AT et al. The American College of Rheumatology 1990 criteria for the classification of Churg-Strauss syndrome (allergic granulomatosis and angiitis). Arthritis and Rheumatism, 1990, 33:1094-1100.

5. Guillevin L et al. Antineutrophil cytoplasm antibodies in systemic polyarteritis nodosa with and without hepatitis B virus infection and Churg-Strauss syndrome-62 patients. Journal of Rheumatology, 1993, 20:1345-1349.

6. Stipić-Marković A et al. Churg-Strauss syndrome: a case report. Acta Clinica Croatica, 2002, 41:95-98.

7. Arm JP, Mark EJ. Case records of the Massachusetts General Hospital. Weekly clinicopathological exercises. Case 30-2000. A 25-year-old man with asthma, cardiac failure, diarrhea, and weakness of the right hand. New England Journal ofMedicine, 2000, 343:953-961.

8. Guillevin L, Pagnoux C, Mouthon L. Churg-Strauss syndrome. New York, Thieme Medical Publishers, 2004:535-545.

9. Pagnoux C, Guilpain P, Guillevin L. Churg-Strauss syndrome. Current Opinion in Rheumatology, 2007, 19:25-32.

10. Taniguchi M et al. Treatment for Churg-Strauss syndrome: induction of remission and efficacy of intravenous immunoglobulin therapy. Allergology International, 2007, 56:97-103.

11. Noth I, Strek ME, Leff AR. Churg-Strauss syndrome. Lancet, 2003, 361:587-594.

12. Lhote F, Cohen P, Guillevin L. Polyarteritis nodosa, microscopic polyangiitis and Churg-Strauss syndrome. Lupus, 1998, 7:238-258.
13. Conron M, Beynon HLC. Rare diseases: Churg-Strauss syndrome. Thorax, 2000, 55:870-877.

14. Patterson R. Allergic bronchopulmonary aspergillosis. Immunology and Allergy Clinics of North America, 1998, 18:471-478.

15. Varkey B. Allergic bronchopulmonary aspergillosis clinical perspectives. Immunology and Allergy Clinics of North America, 1998, 18:479-501.

16. Brezis M, Lafair J. Thrombocytosis in chronic eosinophilic pneumonia. Chest, 1979, 76:231-232.

17. Mayo JR et al. Chronic eosinophilic pneumonia: CT findings in six cases. American journal of roentgenology, 1989, 153(4):727-730.

18. Weller PF, Bubley GJ. The idiopathic hypereosinophilic syndrome. Blood, 1994, 83:2759-2779.

19. Allen JN, Davis WB. Eosinophilic lung diseases. [Am Thoracic Soc. Ref Type: Generic]. American Journal of Respiratory and Critical Care Medicine, 1994, 150:1423-1438.

20. Riccio G et al. Churg-Strauss angiitis. Chest, 1993, 103:16351636.

21. Guillevin L et al. Systemic necrotizing angiitis with asthma: causes and precipitating factors in 43 cases. Lung, 1987, 165:165-172.

22. Phanuphak P, Kohler PF. Onset of polyarteritis nodosa during allergic hyposensitization treatment. American Journal of Medicine, 1980, 68:479-485.

23. Fallah-Tafti $\mathrm{S}$ et al. Churg-Strauss syndrome after introducing oral steroid to inhaler: a report of three cases. Iranian Journal of Allergy, Asthma, and Immunology, 2006, 5:89-94.

24. Kashef $\mathrm{S}$ et al. Churg-Strauss syndrome in an 8-year-old girl. Iranian Journal of Allergy, Asthma, and Immunology, 2004, 3:41-43.

25. Fallah Tafti S, Mokri B, Mansoori N. Churg-Strauss syndrome after polypectomy in asthmatic and allergic patients. Iranian Journal of Allergy, Asthma, and Immunology, 2009, 8:111-115. 